\title{
Soroprevalência da doença de Chagas em crianças em idade escolar do Estado do Espírito Santo, Brasil, em 1999-2000
}

\author{
Chagas' disease seroprevalence \\ among school-age children in Espírito \\ Santo State, Brazil, 1999-2000
}

Paulo Augusto Sessa 1

Ricardo Ramos Pimentel 2

Adelson Luiz Ferreira 1

Aloísio Falqueto 1

\footnotetext{
1 Unidade de Medicina Tropical, Departamento de Patologia, Centro Biomédico, Universidade Federal do Espírito Santo. Av. Marechal Campos 1468 Vitória, ES

29040-090, Brasil. sessapa@escelsanet.com.br 2 Fundação Nacional de Saúde,

Ministério da Saúde. Rua Moacir Strauch 85, Vitória, ES

29055-630, Brasil.

ricpimentel@bol.com.br
}

\begin{abstract}
Although the Brazilian State of Espírito Santo is not considered endemic for Chagas' disease, the sylvatic triatomines occurring there frequently invade houses, increasing the chances of Trypanosoma cruzi transmission to man. The epidemiological pattern of the disease in Espírito Santo was evaluated by a serological survey of 5,243 schoolchildren ages 7 to 14 years, residents of 17 municipalities. Indirect immunofluorescence, indirect hemagglutination, and immunoenzymatic (ELISA) tests were positive in only one person, representing only 0.019\% of the total. This result was similar to those found by other authors in previous studies. Based on the results of serological tests it is concluded that the epidemiological pattern of Chagas' disease in Espirito Santo remains stable, despite the intensive destruction of the Atlantic Forest that has occurred in recent decades.
\end{abstract}

Key words Chagas Disease; Triatominae; Child Health; Seroepidemiologic Studies

Resumo Embora o Estado do Espírito Santo não seja considerado endêmico para a doença de Chagas, triatomíneos silvestres que lá ocorrem freqüentemente invadem as casas, com chances de transmissão da doença às pessoas. Avaliou-se o padrão epidemiológico da moléstia no Estado por meio de um inquérito sorológico realizado em 5.243 escolares de 7 a 14 anos de idade, residentes em 17 municípios. Os testes de Imunofluorescência Indireta, Hemaglutinação Indireta e Imunoenzimático (ELISA) foram positivos em apenas uma pessoa, representando 0,019\% do total. O resultado foi semelhante aos encontrados por outros autores em investigações anteriores. Concluiu-se que, apesar da intensa destruição da Mata Atlântica ocorrida nas últimas décadas, o padrão epidemiológico da doença de Chagas permanece estável, a julgar pelo resultado dos testes sorológicos.

Palavras-chave Doença de Chagas; Triatominae; Saúde Infantil; Estudos Soroepidemiológicos 


\section{Introdução}

No período de 1975 a 1983, como parte do Programa de Controle da Doença de Chagas, o Ministério da Saúde (MS) realizou em quase todo o Brasil, um inquérito sorológico (Camargo et al., 1984) e um inquérito triatomínico (Silveira et al., 1984), com o objetivo de atualizar as informações sobre a prevalência da infecção e sobre a distribuição dos vetores. Dessa forma, foram conhecidas as áreas endêmicas da doença e também definidas e priorizadas as ações de controle.

No Estado do Espírito Santo, esses inquéritos mostraram baixa prevalência da infecção por Trypanosoma cruzi e ausência de espécies de triatomíneos domiciliadas ou semidomiciliadas. Devido a esses resultados, o Espírito Santo foi considerado área de vigilância epidemiológica e nenhuma medida de controle de vetores precisou ser adotada.

Silveira et al. (1984) referiram a ocorrência de quatro espécies de triatomíneos no Estado do Espírito Santo: Triatoma vitticeps, o mais freqüentemente capturado e o de maior distribuição geográfica, Panstrongylus megistus, Triatoma tibiamaculata e Panstrongylus geniculatus. Sessa \& Carias (1986) encontraram taxas de $64,7 \%$ de infecção natural por flagelados morfo- logicamente semelhantes ao Tr. cruzi em T. vitticeps, de $60,0 \%$ em $P$. megistus e de $42,8 \%$ em $P$. geniculatus. Apesar de não haver evidências de domiciliação dessas espécies de triatomíneos (Barros et al., 1975; Dias et al., 1989; Sessa \& Carias, 1986), foi realizado o presente inquérito sorológico com o objetivo de avaliar se houve aumento do número de casos autóctones da doença de Chagas no Estado.

\section{Metodologia}

Durante o período de abril de 1999 a novembro de 2000, foram colhidas em papel filtro Klabin $80 \mathrm{~g}$ amostras de sangue de 5.243 escolares de 7 a 14 anos de idade, residentes na área rural de 17 dos 78 municípios do Estado do Espírito Santo (Tabela 1; Figura 1). A seleção de 16 desses municípios foi por sorteio dentre os 32 cuja população residente na área rural era superior a $50 \%$. O Município de Iconha, embora não atendesse a esse critério, foi incluído no inquérito devido à ocorrência recente de um caso agudo da doença de Chagas (Fundação Nacional de Saúde, dados não publicados).

Todos os eluatos obtidos dos papéis filtro foram submetidos à imunofluorescência indireta

Tabela 1

Resultado do inquérito sorológico para doença de Chagas em escolares de 7 a 14 anos de 17 municípios

do Estado do Espírito Santo, Brasil, realizado no período de abril de 1999 a novembro de 2000.

\begin{tabular}{|c|c|c|c|}
\hline Municípios & Existentes & $\begin{array}{c}\text { Escolares } \\
\text { Examinados(\%) }\end{array}$ & Positivos \\
\hline Afonso Cláudio & 425 & $221(52,00)$ & 0 \\
\hline Águia Branca & 625 & $318(50,88)$ & 0 \\
\hline Alfredo Chaves & 484 & $257(53,09)$ & 0 \\
\hline Atílio Vivacqua & 1.225 & $403(32,89)$ & 0 \\
\hline Divino do São Lourenço & 1.236 & $405(32,79)$ & 1 \\
\hline Domingos Martins & 1.282 & $413(32,31)$ & 0 \\
\hline Ibitirama & 619 & $296(47,81)$ & 0 \\
\hline Iconha & 720 & $325(45,13)$ & 0 \\
\hline Laranja da Terra & 570 & $307(53,85)$ & 0 \\
\hline Marilândia & 465 & $257(55,26)$ & 0 \\
\hline Mimoso do Sul & 935 & $182(19,46)$ & 0 \\
\hline Mucurici & 361 & $153(42,38)$ & 0 \\
\hline Muqui & 657 & $387(58,90)$ & 0 \\
\hline Pancas & 1.338 & $433(32,36)$ & 0 \\
\hline Rio Bananal & 341 & $252(73,90)$ & 0 \\
\hline Santa Leopoldina & 1.211 & $396(32,70)$ & 0 \\
\hline Santa Tereza & 492 & $248(50,40)$ & 0 \\
\hline Total & 12.986 & $5.243(40,37)$ & 1 \\
\hline
\end{tabular}


(IFI), na diluição discriminante de 1:20. Após essa triagem, colheram-se amostras de sangue por punção venosa de todos os escolares com resultados positivos ou duvidosos, para serem submetidas à IFI, à hemaglutinação indireta (HI) e ao Enzime-Linked ImmunoSorbent Assay (ELISA). Na confirmação desses resultados participaram o Laboratório Central da Secretaria de Saúde do Estado do Espírito Santo e o Instituto Octavio Magalhães da Fundação Ezequiel Dias. Foram considerados positivos os soros reagentes em pelo menos dois testes diferentes, como preconiza o MS (1996). Os antígenos usados na IFI foram o T. cruzi Bio-Manguinhos (FIOCRUZ) e o Imunocruzi (BIOLAB); no ELISA foi utilizado o EIE-recombinante-Chagas Bio-Manguinhos e na HI foi o Hemacruzi (BIOLAB).

Dos coabitantes dos escolares com resultados positivos ou duvidosos, colheram-se amostras de sangue em papel filtro para serem submetidas à IFI.

A metodologia empregada obedeceu às normas técnicas para avaliação do Programa de Controle da Doença de Chagas do MS (1994) e a rotina de trabalho de campo da Fundação Nacional de Saúde. Assim, a taxa de escolarização de cada município selecionado, obtida nas respectivas secretarias de educação, era superior a $80 \%$. Todas as escolas rurais foram incluídas no trabalho. O consentimento para as crianças participarem do inquérito foi obtido dos pais nas reuniões realizadas nas escolas. Para o cálculo do tamanho da amostra, levou-se em conta o número de escolares da zona rural de cada município e a estimativa da população infectada pelo Tr. cruzi, na faixa estudada, de acordo com o Inquérito Nacional (1976-1980). Escolheu-se trabalhar com a proporção de 41 a $50 \%$, ao invés de zero a $10 \%$, para obter-se uma amostra maior. O número de crianças amostrado em cada escola foi calculado segundo fórmula constante nessas normas técnicas. Em cada escola, os alunos foram selecionados por sorteio até que fosse completado o total necessário. A colheita de sangue em papel filtro foi realizada nas escolas e a de sangue por punção venosa foi realizada nos domicílios.

Os resultados do inquérito foram encaminhados às prefeituras, que se responsabilizaram por repassá-los aos órgãos de saúde locais, escolas e comunidades. Apenas as crianças positivas receberam o resultado individual em suas casas.
Figura 1

Municípios do Estado do Espírito Santo, Brasil, onde foi realizado inquérito sorológico entre escolares, no período de abril de 1999 a novembro de 2000.
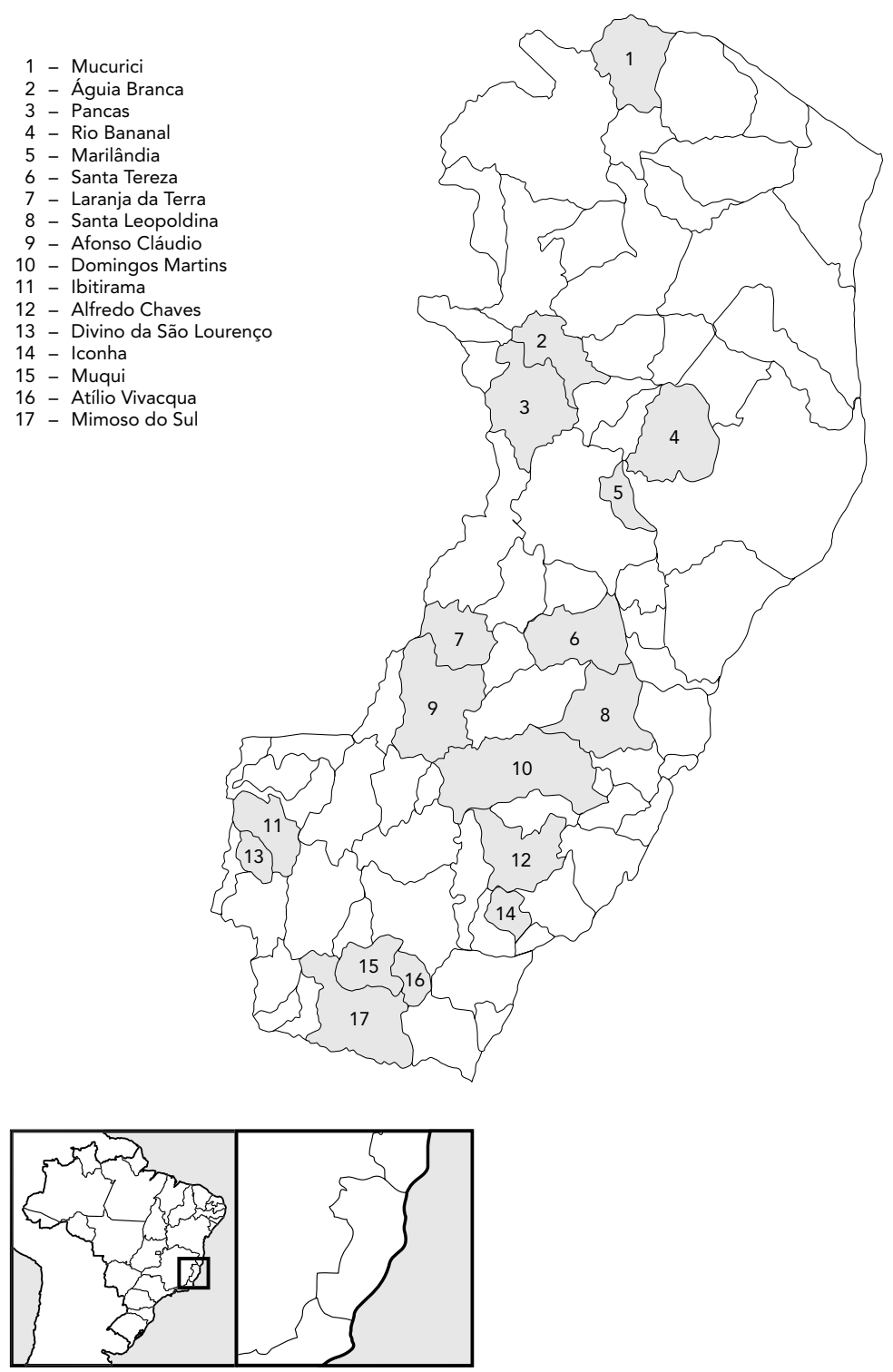


\section{Resultados}

A IFI resultou positiva ou duvidosa em 42 das 5.243 amostras de sangue dos escolares, colhidas em papel filtro. Posteriormente, a IFI, a HI e o ELISA realizados nos soros desses 42 escolares só confirmaram positividade em um, o que representou $0,019 \%$ do total (Tabela 1). Essa criança tinha 12 anos de idade, era do sexo masculino e residia na localidade de Córrego Azul, Município de Divino de São Lourenço. Foi positivo na IFI no título de 1:40 e positivo no teste de ELISA. O exame clínico e os exames complementares não revelaram qualquer alteração orgânica devida à doença de Chagas ou a outra doença infecciosa. Nesse município não há relatos de ocorrência de leishmaniose visceral e são raros os casos de leishmaniose tegumentar.

Todos os coabitantes desses 42 escolares tiveram resultados negativos na IFI.

\section{Discussão}

A doença de Chagas autóctone parasitologicamente comprovada no Estado do Espírito Santo foi pela primeira vez demonstrada por Barros et al. (1975). Esses autores examinaram soros de 3 mil escolares da zona rural de 50 dos $53 \mathrm{mu}-$ nicípios do Estado por meio da IFI, da HI e da reação de fixação de complemento, e encontraram apenas dois escolares reagentes nos três testes e que também foram positivos no xenodiagnóstico $(0,066 \%)$.

O inquérito sorológico executado pelo MS (Camargo et al., 1984) veio confirmar os achados de Barros et al. (1975). Por meio da IFI, foram examinadas amostras de sangue colhidas em papel filtro da população geral residente fora das áreas urbanas de todos os municípios do Estado do Espírito Santo, a exceção da capital, Vitória. A prevalência de resultados positivos foi de $0,32 \%$. De acordo com dados adicionais desse inquérito, fornecidos pelo MS e não publicados, a prevalência de resultados positivos na faixa etária de 7 a 14 anos foi $0,058 \%$, e a de resultados positivos entre autóctones da população geral foi $0,09 \%$.
Além desses inquéritos, apenas seis casos autóctones no Estado foram objetos de publicação, e todos diagnosticados em ambulatórios de clínica médica de hospitais de Vitória (Peçanha et al., 1983; Pinto et al., 1969, 1986; Santos et al., 1969).

O resultado do presente inquérito é semelhante aos encontrados por Barros et al. (1975), e pelo MS (Camargo et al., 1984), e demonstrou que a situação da doença de Chagas no Estado do Espírito Santo não se alterou. Nos poucos casos autóctones registrados, a transmissão deveu-se, provavelmente, a adultos de T. vitticeps, espécie de hábito silvestre mais capturada nos domicílios do Estado (Dias et al., 1989; Sessa \& Carias, 1986; Silveira et al., 1984). Ocasionalmente são capturadas também ninfas dessa espécie no interior dos domicílios, as quais encontram-se geralmente no mesmo estádio de evolução (Fundação Nacional de Saúde, dados não publicados), sugerindo posturas isoladas de fêmeas procedentes do ambiente silvestre e não a instalação definitiva de colônias no intradomicílio.

T. vitticeps apresenta elevadas taxas de infecção natural pelo Tr. cruzi e, entre os exemplares capturados no domicílio, a maioria apresenta sangue humano no intestino (Dias et al., 1989). Apesar disso, essa espécie é considerada um mau vetor pois não costuma defecar durante ou imediatamente após a alimentação, e coloniza mal os ecótopos artificiais (Dias et al., 1989; Gonçalves et al., 1988, 1998, 2000; Sessa \& Carias, 1986).

\section{Conclusão}

A prevalência da doença de Chagas autóctone no Estado do Espírito Santo se mantém baixa. A ocorrência de casos humanos parece depender da invasão de domicílios por triatomíneos silvestres adultos, em especial T. vitticeps. A possibilidade de mudança de hábitos desses triatomíneos em virtude da grande devastação da Mata Atlântica ocorrida nas últimas décadas, reafirma a necessidade de se manter uma vigilância sobre a situação epidemiológica dessa doença no Estado. 


\section{Referências}

BARROS, G. C.; MAYRINK, W.; SALGADO, A. A.; BARROS, R. C. G. \& SESSA, P. A., 1975. Contribuição para o conhecimento da doença de Chagas autóctone no Estado do Espírito Santo. Revista do Instituto de Medicina Tropical de São Paulo, 17:319-329.

CAMARGO, M. E.; SILVA, G. R.; CASTILHO, E. A. \& SILVEIRA, A. C., 1984. Inquérito sorológico da prevalência da infecção chagásica no Brasil, 1975/ 1980. Revista do Instituto de Medicina Tropical de São Paulo, 26:192-204.

DIAS, J. C. P.; FEITOSA, V. R.; FERRAZ FILHO, N. A.; RODRIGUES, V. L. C.; ALENCAR, A. S. \& SESSA, P. A., 1989. Fonte alimentar e potencial vetorial de Triatoma vitticeps (Stal, 1859) com relação à doença de Chagas humana no Estado do Espírito Santo, Brasil (Hemiptera, Reduviidae). Memórias do Instituto Oswaldo Cruz, 84:165-173.

GONÇALVES, T. C. M.; VICTÓRIO, V. M. N.; JURBERG, J. \& CUNHA, V., 1988. Biologia do Triatoma vitticeps (Stal, 1859) em condições de laboratório (Hemiptera: Reduviidae: Triatominae). I. Ciclo evolutivo. Memórias do Instituto Oswaldo Cruz, 83:519523.

GONÇALVES, T. C. M.; OLIVEIRA, E.; DIAS, L. S.; ALMEIDA, M. D.; NOGUEIRA, W. O. \& PIRES, F. D. A., 1998. An investigation on the ecology of Triatoma vitticeps (Stal, 1859) and its possible role in the transmission of Trypanosoma cruzi, in the locality of Triunfo, Santa Maria Madalena municipal district, State of Rio de Janeiro, Brazil. Memórias do Instituto Oswaldo Cruz, 93:711-717.

GONÇALVES, T. C. M.; ROCHA, D. S. \& CUNHA, R. A., 2000. Feeding patterns of Triatoma vitticeps in the State of Rio de Janeiro, Brazil. Revista de Saúde Pública, 34:348-352.

MS (Ministério da Saúde), 1994. Inquérito Sorológico para Avaliação do Programa de Controle da Doença de Chagas - Normas Técnicas. Brasília: Fundação Nacional de Saúde, MS.
MS (Ministério da Saúde), 1996. Controle da Doença de Chagas - Diretrizes Técnicas. Brasília: Fundação Nacional de Saúde, MS.

PEÇANHA, P. M.; SESSA, P. A.; SILVA, F. J. D.; PEREIRA, R. S. \& ALMEIDA, I. M., 1983. Doença de Chagas apresentação de três casos autóctones do Estado do Espírito Santo. In: XX Congresso da Sociedade Brasileira de Medicina Tropical, Resumos, p. 33. Rio de Janeiro: Sociedade Brasileira de Medicina Tropical.

PINTO, A. F. S.; SANTOS, U. M.; MURAD, V.; PEREIRA, F. E. L.; ZAGANELLI, F. L. \& ALMEIDA, Z., 1969. Doença de Chagas no Estado do Espírito Santo. V - Primeiro caso de Chagas diagnosticado no Município de Alfredo Chaves, ES. Revista da Sociedade Brasileira de Medicina Tropical, 3:53.

PINTO, L. F. S.; VIEIRA, N. E. R.; MILANEZ, M. C.; PEREIRA, F. E. L. \& SESSA, P. A., 1986. Doença de Chagas autóctone no E. Santo: Relato de caso agudo em gestante com transmissão para o feto. Revista da Sociedade Brasileira de Medicina Tropical, 19: 78.

SANTOS, U. M.; MURAD, V.; CHAPADEIRO, E. \& PEREIRA, F. E. L., 1969. Doença de Chagas no Estado do Espírito Santo. IV - Primeiro caso diagnosticado no Município de Rio Novo do Sul. Revista da Sociedade Brasileira de Medicina Tropical, 3:52-53.

SESSA, P. A. \& CARIAS, V. R. D., 1986. Infecção natural de triatomíneos do Espírito Santo por flagelados morfologicamente semelhantes ao Trypanosoma cruzi. Revista da Sociedade Brasileira de Medicina Tropical, 19:99-100.

SILVEIRA, A. C.; FEITOSA,V. R. \& BORGES, R., 1984 Distribuição de triatomíneos capturados no ambiente domiciliar, no período 1975/83, Brasil. Revista Brasileira de Malariologia e Doenças Tropicais, 36:15-312.

Recebido em 14 de novembro de 2001

Versão final reapresentada em 3 de junho de 2002

Aprovado em 24 de junho de 2002 